\title{
Uso da fluoxetina no tratamento da tricotilomania felina
}

\author{
Use of fluoxetine in the treatment of feline psycogenic alopecia
}

\author{
Marlos Gonçalves Sousa ${ }^{1}$ Lilian Stefanoni Ferreira ${ }^{2}$ \\ Daniel Guimarães Gerardi ${ }^{3}$ Mirela Tinucci Costa ${ }^{4}$
}

\section{- RELATO DE CASO -}

\section{RESUMO}

A tricotilomania ou alopecia psicogênica felina é uma dermatopatia de origem psicogênica, decorrente da lambedura compulsiva do pelame, realizada pelo gato em situações de estresse. Tal distúrbio decorre de alterações neurohormonais e pode associar-se à introdução de novos animais el ou crianças no ambiente. Além de mudanças de manejo e atitude para com o animal, sugere-se o emprego de ansiolíticos no tratamento da doença. A fluoxetina foi utilizada no tratamento de cinco gatos domésticos com tricotilomania, apresentando inibição do comportamento de lambedura, com repilação após dois a três meses de terapia.

Palavras-chave: distúrbio neuro-hormonal - alopecia psicogênica - dermatopatia.

\section{ABSTRACT}

Feline psycogenic alopecia occurs when cats lick their hair compulsively. Stress situations play a role in this disturbance, due to changes in neurohormone mechanisms. The introduction of a new animal or baby in the household can also be associated. Besides behavioral changes towards the animal, the use of anxiolytics is suggested to treat the disease. Fluoxetine was used in the treatment of 5 domestic cats with psycogenic alopecia, showing inhibition of licking attitude, with repilation within two to three months of use.

Key words: feline neurohormone disturbances - psycogenic alopecia - dermatologic disease.

\section{INTRODUÇÃO}

$\mathrm{Na}$ dermatologia humana, acredita-se que os fatores emocionais nas doenças cutâneas tenham grande significância. Cita-se que, caso sejam ignorados esses fatores, o tratamento eficaz seria impossível em pelo menos $40 \%$ dos pacientes (PANCONESI, 1984).

Tanto em animais como em seres humanos, sabe-se que o sistema nervoso central pode modular a resposta imune e o prurido por meio de mecanismos neuro-hormonais (PANCONESI, 1984; SCOTT et al., 1996). Na medicina veterinária, o papel exato dos neurotransmisores neste processo ainda é incerto (SCOTT et al., 1996).

Em seres humanos, as condições psicodermatológicas dividem-se em: distúrbios psicogênicos (distúrbio cutâneo exacerbado por estresse emocional); distúrbios psiquiátricos primários (ansiedade, depressão, alucinação e comportamento obsessivo-compulsivo) e distúrbios psiquiátricos secundários (KOO \& PHAM, 1992). Em veterinária, existe evidência de que os distúrbios psicogênicos sejam a causa das dermatoses psicogênicas, cujas lesões seriam auto-induzidas (OVERALL, 1992; RAPOPORT et al., 1992). Solidão, falta de atenção, hiperatividade e ansiedade são condições comportamentais que podem estar presentes nos animais afetados (SCOTT et al., 1996).

Nos felinos, a lambedura excessiva dos pêlos pode ser resultante de ansiedade de cunho nervoso. Esse comportamento crônico, excessivo e estereotipado, resulta em áreas de alopecia (WALTON, 1986; WILLEMSE et al., 1989; SCOTT et al., 1996). Ocasionalmente, pode haver uma causa concomitante, como atopia, dermatofitose, hipersensibilidade

${ }^{1}$ Médico Veterinário, Mestrando em Clínica Médica, Faculdade de Ciências Agrárias e Veterinárias (FCAV), Universidade Estadual paulista (UNESP), Campus de Jaboticabal, Via de Acesso Prof. Paulo Donato Castellane, S/N, Campus da UNESP, Jaboticabal, SP. E-mail: marlos98@yahoo.com. Autor para correspondência.

${ }^{2}$ Médico Veterinário, Residente em Clínica Médica de Pequenos Animais, Hospital Veterinário “Governador Laudo Natel”, FCAV, UNESP, Jaboticabal.

${ }^{3}$ Médico Veterinário, Mestrando em Clínica Médica, FCAV, UNESP, Campus de Jaboticabal.

${ }^{4}$ Professor Doutor, Departamento de Clínica e Cirurgia Veterinária, FCAV, UNESP, Campus de Jaboticabal. 
alimentar ou dermatite alérgica a picada de pulga (WILKINSON \& HARVEY, 1996). Entretanto, essa não é a etiologia habitual e acredita-se que a causa principal da ansiedade sejam fatores psicológicos, incluindo deslocamento, introdução de novo animal ou bebê na casa, novos móveis ou mudança de posição do mobiliário antigo, mudança do lugar do comedouro e das bandejas sanitárias, viagem, hospitalização, competição e invasão ou perda de território (WALTON, 1986; SCOTT et al., 1996; WILKINSON \& HARVEY, 1996).

Nos animais submetidos a estresse, supõese que ocorra um aumento dos níveis dos hormônios indutor dos melanócitos e adrenocorticotrópico, levando a uma maior produção de endorfinas, as quais podem gerar o comportamento anormal de lambedura, devido ao seu efeito narcótico (WILLEMSE et al., 1989; SCOTT et al., 1996).

$\mathrm{Na}$ alopecia psicogênica felina ou tricotilomania, o animal lambe e masca os pêlos em regiões que podem ser alcançadas facilmente, tais como a face medial dos membros pélvicos, as regiões abdominal ventral e lombossacra dorsal. Tal comportamento resulta em alopecia bilateral simétrica (WILKINSON \& HARVEY, 1996), sendo o diagnóstico da dermatose psicogênica comumente obtido por exclusão de outras enfermidades tegumentares (WILKINSON \& HARVEY, 1996).

Sabe-se que, se os problemas que levaram ao desenvolvimento do distúrbio puderem ser removidos, pode-se evidenciar melhora em cerca de 30 dias com o uso de ansiolíticos (SCOTT et al., 1996). Dentre os fármacos citados para o tratamento, incluemse fenobarbital, diazepam (SCOTT et al., 1996), primidona (HOLZWORTH, 1987), naloxona (WILLEMSE et al., 1989) e fluoxetina (SOUSA et al., 2002). Além disso, colocando-se um colar de contenção cervical (tipo elizabetano) no animal, poderá ser observada repilação em áreas que não podem ser lambidas (SCOTT et al., 1996).

A fluoxetina é um fármaco antidepressivo, pertencente ao grupo dos inibidores seletivos da captação de serotonina. Sua ação decorre do aumento da concentração de serotonina pelo sistema nervoso central, devido ao bloqueio pré-sináptico neuronal dos receptores de serotonina 5-HT (5hidroxitriptamina). É indicada em distúrbios compulsivos, automutilação, ansiedade e agressividade (ANDRADE et al., 2002).

Este trabalho tem por objetivo, descrever cinco casos de tricotilomania felina, os quais foram tratados utilizando-se o cloridrato de fluoxetina, com bons resultados.

\section{RELATO DOS CASOS}

Foram atendidos pelo Serviço de Clínica Médica de Pequenos Animais, do Hospital Veterinário da UNESP, Campus de Jaboticabal-SP, entre Novembro de 2001 e Agosto de 2002, cinco felinos domésticos, trazidos individualmente, sendo dois machos e três fêmeas, com idades variando entre três e seis anos.

Em todos os casos, o histórico clínico era semelhante, com o proprietário queixando-se de perda de pêlos progressiva ao longo da região abdominal lateroventral e nas faces internas e externas dos membros pélvicos (Figura 1-A).

A anamnese permitiu constatar que houve introdução de bebê na casa previamente ao desenvolvimento da alopecia em um caso; entrada de novos animais no mesmo espaço em dois casos; e reforma do quintal e gatil nos demais dois casos. Dentre os cinco casos, apenas três proprietários notaram exacerbação dos cuidados de higiene e lambedura por parte dos felinos. Segundo as informações coligidas, todos os animais apresentavam adequada ingestão de água e alimento, e aparentemente não havia competição pelo alimento entre os contactantes.

Ao exame físico, verificou-se completa normalidade dos parâmetros clínicos. A inspeção dermatológica relevou pêlos dilacerados, com aspecto tonsurado nas áreas afetadas. Entretanto, não se observou qualquer sinal de processo inflamatório e/ ou séptico da pele. Ao se tentar avulsionar os pêlos das regiões afetadas e daquelas não-afetadas, os mesmos apresentavam aderência semelhante, não se desprendendo com facilidade.

Visando estabelecer o diagnóstico, foi realizado raspado de pele superficial e profundo, em três pontos distintos da pele, em quatro dos casos. Foi solicitada observação microscópica das amostras preparadas. Todos os animais foram submetidos à inspeção dermatológica indireta com a lâmpada de Wood, não sendo evidenciando qualquer sinal de fluorescência.

Os exames parasito e micológico diretos do raspado cutâneo revelaram ausência de ácaros, hifas ou esporos fúngicos. Entretanto, pôde-se notar evidência de traumatismo nas hastes pilosas, sendo bem visíveis suas extremidades dilaceradas (mordidas), diferentemente constatado no tricograma de áreas não afetadas, que exibiu extremidades levemente afiladas.

Por exclusão de outras dermatopatias, estabeleceu-se o diagnóstico clínico de alopecia 


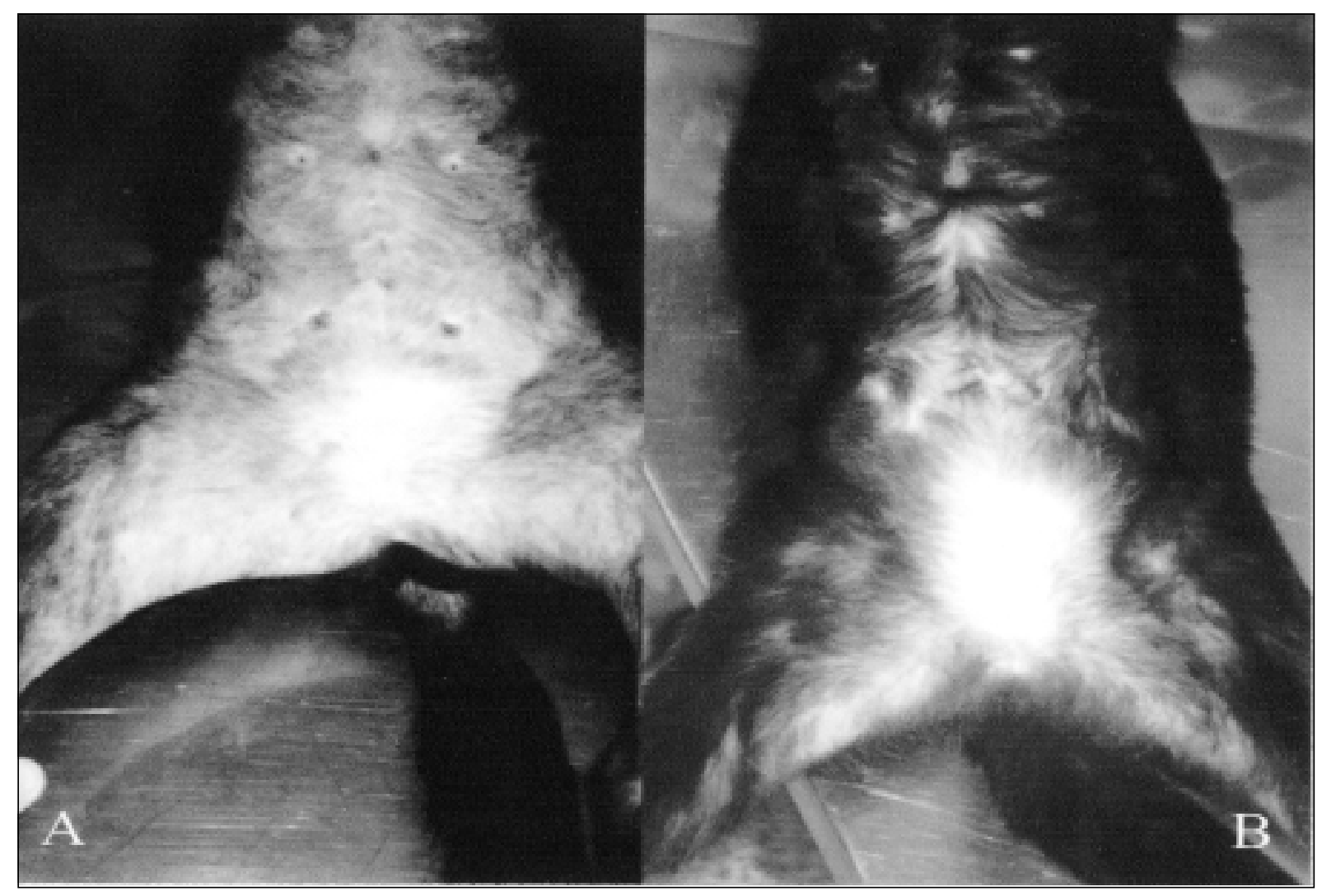

Figura 1 - Aspecto da região abdominal ventral de um dos felinos com tricotilomania. Nota-se área alopécica, com pele íntegra, sem sinais de inflamação (A) visualizada durante atendimento inicial. Em (B) observa-se o mesmo animal 60 dias após o início do uso do cloridrato de fluoxetina, já evidenciando repilação significativa da área previamente acometida.

psicogênica felina ou tricotilomania, baseando-se nos achados do tricograma (em quatro felinos) e no histórico da anamnese (nos cinco felinos).

Diante do diagnóstico clínico firmado, decidiu-se estabelecer o tratamento com o cloridrato de fluoxetina em um dos casos atendidos. Associado ao medicamento, o proprietário foi alertado da necessidade de se intensificar o tempo e cuidados a dispensar ao animal, dedicando-lhe mais atenção, visto que o distúrbio desencadeou-se após a introdução de um novo felino no ambiente.

A fluoxetina foi prescrita na dose de $1 \mathrm{mg} /$ $\mathrm{kg}$, duas vezes ao dia, por via oral. O animal foi reavaliado 30 dias após o início do tratamento. No retorno, foi observada melhora significativa, com notável início do crescimento piloso nas áreas afetadas. Sessenta dias após a prescrição do tratamento, o animal ainda recebia o medicamento e já apresentava repilação quase que total, sendo difícil identificar as áreas acometidas preteritamente (Figura 1-B).

$A$ adequada resposta à terapia apresentada pelo paciente foi o fator determinante para se reempregar o cloridrato de fluoxetina, associado à indicação de mudança de atitude em relação ao animal, nos outros quatro casos subseqüentes. Em todos eles, observou-se melhora completa em até três meses de uso do fármaco. Especificamente em um dos animais, o medicamento continuou sendo fornecido na dose de $0,5 \mathrm{mg} / \mathrm{kg}$, uma vez ao dia, visto que após sua retirada, ele voltou a desenvolver lambedura compulsiva, embora o proprietário tenha informado que também houve mudança de atitude em relação ao animal.

\section{DISCUSSÃO}

Os cinco casos de tricotilomania descritos desenvolveram-se após situações que desencadearam alterações comportamentais nos felinos, resultando em estresse para os mesmos, assim como descrito por WALTON (1986) e WILLEMSE et al., (1989).

Tal como citado por SCOTT et al. (1996), a alopecia estendia-se pelas regiões passíveis de serem alcançadas pelos felinos, que as lambiam compulsivamente, caracterizando comportamento 
estereotipado. Desse modo, as áreas afetadas distribuíam-se pela região abdominal ventral e faces internas e externas dos membros pélvicos, locais estes também referidos por WILKINSON \& HARVEY (1996).

Por tratar-se uma dermatose psicogênica, a tricotilomania tem seu diagnóstico firmado por exclusão de outras causas de alopecia e prurido, associado ao histórico clínico do paciente. Assim como citado por WILKINSON \& HARVEY (1996) e adotado por outros autores, nos cinco casos atendidos, o diagnóstico foi firmado baseando-se na anamnese e no descarte de outras dermatopatias.

O cloridrato de fluoxetina foi empregado empiricamente no primeiro caso de tricotilomania, verificando-se adequada resposta terapêutica (SOUSA et al., 2002). Tendo em vista o bom resultado obtido, seu uso foi estendido aos demais casos, obtendo-se, também, excelente resposta clínica, com repilação das áreas alopécicas.

Entretanto, em todos os casos, o proprietário foi, também, instruído sobre a necessidade de mudança de manejo e atitude com relação ao animal, conforme preceituam SCOTT et al. (1996).

Embora SCOTT et al. (1996) tenham observado melhora dos animais em cerca de 30 dias após a introdução do ansiolítico, nos cinco casos em tela, o tempo de uso variou de dois a três meses, com necessidade de perpetuação da medicação em apenas um dos animais para se obter a completa resolução do problema.

\section{CONCLUSÃO}

De acordo com o resultado obtido no tratamento de cinco casos de alopecia psicogênica felina ou tricotilomania, pode-se inferir que o cloridrato de fluoxetina, na dose de $1,0 \mathrm{mg} / \mathrm{kg}$, duas vezes ao dia, é um fármaco eficaz para o tratamento dessa dermatose psicogênica. Além disso, ressalta-se ainda, a inexistência de qualquer efeito adverso no curso dos tratamentos ora citados.

\section{REFERÊNCIAS BIBLIOGRÁFICAS}

ANDRADE, S.F. et al. Terapêutica do sistema nervoso. In: ANDRADE, S.F. Manual de terapêutica veterinária. 2.ed. São Paulo : Roca, 2002. Cap.17. p.347-435.

HOLZWORTH, J. Diseases of the cat: medicine and surgery. Philadelphia : Saunders, 1987. V.1.

KOO, J.Y.M.; PHAM, C.T. Psychodermatology. Practical guidelines in pharmacotherapy. Arch Dermatol, v.128, p.381388, 1992.

OVERALL, K.L. Recognition, diagnosis and management of obsessive-compulsive disorders. Part 1: a rational approach. Canine Pract, v.17, n.2, p.40-44, 1992.

PANCONESI, E. Stress and skin diseases: psychosomatic dermatology. In: PARISH, L.C. Clinics in dermatology. Philadelphia : Lippincott, 1984. p.1-283.

RAPOPORT, J.L.; RYLAND, D.H.; KRIETE, M. Drug treatment of canine acral lick: an animal model of obsessivecompulsive disorder. Arch Gen Psychiatr, v.49, n.7, 517-521, 1992.

SCOTT, D.W.; MILLER, W.H.; GRIFFIN, C.E. Dermatoses psicogênicas. In: ___. Dermatologia de pequenos animais. 5.ed. Rio de Janeiro : Interlivros, 1996. Cap.14. p.790-802.

SOUSA, M.G. et al. Tricotilomania felina - aspectos clínicoterapêuticos. In: MOSTRA CIENTÍFICA DA FMVZ-UNESP, 6 , 2002, Botucatu, SP. Anais... Botucatu : Faculdade de Medicina Veterinária e Zootecnia, 2002. p.80.

WALTON, D.K. Psycodernatoses. In: KIRK, R.W. Current veterinary therapy. 9.ed. Philadelphia : Saunders, 1986. p.557559.

WILLEMSE, T. et al. Feline psycogenic alopecia and the role of the opioid system. In: VON TSCHARNER, C.; HALLIWELL, R.E.W. Advances in veterinary dermatology. London : Baillière Tindall, 1989. p.195-198.

WILKINSON, G.T.; HARVEY, R.G. Dermatoses psicogênica. In: . Atlas colorido de dermatologia dos pequenos animais. 2.ed. São Paulo : Manole, 1996. Cap.16, p.255-258. 